\title{
Angular Momentum Loss in Polars
}

\author{
Belinda Kalomeni ${ }^{1,2}$ \\ ${ }^{1}$ Department of Physics, İzmir Institute of Technology, 35430 İzmir, Turkey \\ ${ }^{2}$ Visiting Fellow, Institute of Astronomy, University of Cambridge, Madingley Road, \\ Cambridge CB3 0HA, UK
}

\begin{abstract}
We discuss the possible angular momentum loss mechanisms in AM Her type cataclysmic variables and their corresponding mass loss rates using the observed physical parameters of them.
\end{abstract}

Keywords. Stars: binaries, Stars: cataclysmic variables

\section{Introduction}

AM Her binaries as a subclass of magnetic cataclysmic variables consist of a magnetic white dwarf primary and a mass loosing late-type main-sequence secondary star. High magnetic field intensities determine most of the observed properties of them. A number of methods, successive cyclotron harmonics, zeeman splitting and polarization have been used to determine it. Cyclotron spectra of polars have been the subject of many studies (Campbell et al. 2008, Kalomeni et al. 2005a and references therein). Polars show short term and long term light variations (Kalomeni et al. 2005b, Kalomeni \& Yakut 2008 and references therein). The knowledge of fundamental parameters of binary stars is of importance for formation and evolution studies of them to determine their possible evolutionary tracks (see King et al. 1994, Baraffe \& Kolb 2000). For a detailed review of CVs we refer the reader to Cropper (1990) and Warner (1995). We collated the basic physical parameters of totally 90 polars presented in literature. They have been studied by numerous authors and the parameters were deduced using different techniques. The collected data and the results will be presented in details in Kalomeni et al. (2009).

\section{Angular Momentum Loss}

The effect of angular momentum loss (AML) in a short period binary star evolution was discussed in details in many papers (e.g., Paczyński 1967, Chau 1978, Verbunt \& Zwaan 1981, Patterson 1984, Yakut et al. 2008). We assumed that the AML driven by magnetic braking (MB) and gravitational radiation (GR) will result in AML from the binary (see Verbunt \& Zwaan 1981). Angular momentum variation due to the stellar activity of the secondary is obtained from Verbunt \& Zwaan (1981) and those for the GR subtracted from Peters (1964). We use Eggleton's (1983) Roche lobe formula to measure the radius of the component. The details of the calculations can be found in Kalomeni et al. (2009). AML rate as a function of orbital period and mass ratio are displayed in Fig. 1a. The Figure shows that at orbital periods $\mathrm{P}>1.7 \mathrm{hr}$ the effects of GR in AML are negligible therefore, we assume that the evolution of systems is driven mainly by MB. While at orbital periods $\mathrm{P} \leqslant 1.7 \mathrm{hr}$ the orbital AML is driven mainly by GR. Knowing the AML rate, along with the parameters compiled, we could deduce the mass loss rate for each mechanism. For the known masses and orbital period the variation of the $\dot{\mathrm{M}}_{2}$ due to the GR and MB with respect to the orbital period and mass ratio are shown in Fig. 1b. 

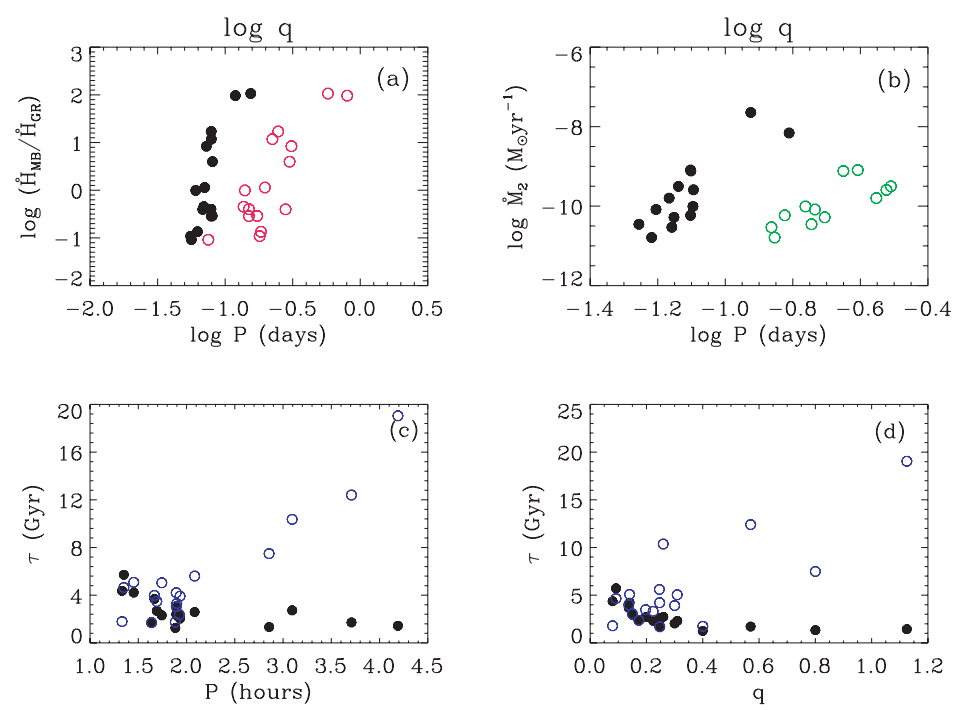

Figure 1. (a) $\dot{\mathrm{H}}_{\mathrm{MB}} / \dot{\mathrm{H}}_{\mathrm{GR}}$ vs orbital period (filled circles) and mass ratio (open circles), (b) the relation between $\dot{\mathrm{M}}_{2}$ and orbital period (filled circles) and mass ratio (open circles), (c) MB (filled circles) and GR (open circles) time scales vs orbital period and (d) MB and GR time scales vs mass ratio.

Extensive studies on polars show that mass accretion rate vary over time. A review for the causes in mass accretion rate variation given by Howell (2004). In Fig. 1c-d MB and GR times scales are shown as a function of orbital period and mass ratio.

\section{References}

Baraffe I. \& Kolb U. 2000, MNRAS, 318, 354

Campbell, R. K., Harrison, T. E., Schwope, A. D., \& Howell, Steve B. 2008, ApJ, 672, 531

Chau, W. Y. 1978, ApJ, 219, 1038

Cropper, M. 1990, SSRv, 54, 195

Eggleton, P. P. 1983, ApJ, 268, 368

Howell, S. B. 2004, ASPC, 315, 353

Kalomeni, B., Pekünlü, E. R., \& Yakut, K. 2005a, A\&SA, 439, 823

Kalomeni, B., Pekünlü, E. R., \& Yakut, K. 2005b, ApESSS, 296, 477

Kalomeni, B. \& Yakut, K. 2008, AJ, 136, 2367

Kalomeni, B. et al. 2009, in preparation

King, A. R., Kolb, U., de Kool, M., \& Ritter, H. 1994, MNRAS, 269, 907

Paczyński, B. 1967, AcA, 17, 287

Patterson, J. 1984, ApJS, 54, 443

Peters, P. C. 1964, PhRv, 136, 1224

Verbunt, F. \& Zwaan, C. 1981, A\&A, 100L, 7

Warner, B. 1995, Cataclysmic Varible Stars, Cambridge Astrophysics Series

Yakut, K., Kalomeni, B., \& Tout, C. A. 2008, mqw, conf, 65 